\title{
PARTICIPATION OF ALLIED HEALTH PROFESSIONALS IN THE PRACTICE OF ANAESTHESIA: REPORT OF A STUDY
}

\author{
J.W.R. MCINTYRE ${ }^{\circ}$
}

\section{INTRODUCTION}

There ARE MANY COUNTRIES where persons that have not graduated from a medical school actively participate in the administration of anaesthesia for surgery. This participation ranges from complete responsibility for the anaesthesia in a location where skilled help is not immediately available to observing simple physiological and mechanical functions with a physician anaesthetist in close proximity. This variety of activities makes the selection of a title for these persons difficult, and there are often considerable differences in the responsibilities of people that have similar titles such as nurse anaesthetists, anaesthesia technician, and so on. It is only with advancing knowledge that serious and partially successful attempts have been made to ensure that all anaesthetics are administered by physicians. However, in some parts of the world this effort has been thwarted by a lack of suitable personnel and a need for economy. It has been claimed that techniques of anaesthesia and the predictability of events during anaesthesia are such that once anaesthesia has been established by a physician specialist in anaesthesia, supervision of the patient can and should be delegated to appropriately trained nonphysician personnel while the physician remains available for immediate action in the operating room. This would enable a department to provide a more flexible service function without an increase in the number of physician anaesthetists. It was with these thoughts in mind that it was decided to evaluate a situation in which, after three months initial training, recently graduated nurses monitored patients once surgical anaesthesia had been established by a specialist physician anaesthetist. The aspects of particular interest were the safety of patients, the integration of the nurses with other personnel in the operating room, the opinions of physician anaesthetists about this new situation, the opinions of nurses about this type of work, and anything else of interest that occurred.

\section{METHOD}

Initially the project was discussed and approved by the Alberta Division of the Canadian Anaesthetists' Society. The nurses continued to be employed by the hospital though largely supported by the funding agency, and legal protection for the participating physicians was available from a comprehensive policy normally held by the University on behalf of its faculty members.

The project was advertised within the hospital and four girls selected from the

'Department of Anaesthesia, University Hospital, Edmonton, Alberta. Supported by the Department of National Health and Welfare, Project No. 609-20-8. 
small number that applied. The criteria used for selection of suitable candidates were: relatively recent graduation; negligible operating room or intensive care experience; an expressed interest in the project; and a claim to some mechanical capability.

The work consisted of assessing the physiological functions customarily observed by anaesthetists during established anaesthesia, and seeing that intravenous infusions and the mechanical equipment commonly associated with the administration of anaesthetics continued to function properly. They were not empowered to administer intravenous drugs or alter the quantity of a drug being administered by inhalation. Thus the decisions they had to make were whether their observations indicated that a physician anaesthetist should be called to administer drugs or adjust equipment. In this way they were in part responsible for assessing the anaesthetic requirements of the patient and the requirements for the patient's homeostasis as the surgery progressed. In the event that the patient's ventilation suddenly became inadequate they were instructed to initiate another method of ventilation while the physician anaesthetist was being sent for. The techniques of general anaesthesia they supervised included commonly employed agents and circuits, but they were not exposed to procedures involving induced hypothermia, hypotension or cardiopulmonary bypass. The patients with which they were supposed to become accustomed were ASA risk categories 1 and 2 who were undergoing general surgical, gynaecological, or orthopedic operations.

Instruction did not include formal lectures. A period of three months was spent in the operating rooms obtaining practical instruction from physician anaesthetists, supplemented by suitable reading. During this time arrangements were made for informal feedback from the operating room supervisor regarding problems that might arise and the four nurses met formally once a week with the project director.

When the three-month training period had been completed a three-month evaluation period began immediately. During this time the nurse was left to look after the patient once surgical anaesthesia had been established to the satisfaction of the physician anaesthetist. The information collected on a form for each patient indicated:

(i) The frequency of casual visits to the operating room; whether or not the anaesthetists adjusted the anaesthesia in any way; what he or she did and the urgency of any change that was made.

(ii) The frequency of visits to the operating room that were requested by the nurse, whether or not the anaesthetist made any adjustments, the nature of these adjustments, and their urgency.

Subsequently the number of intravenous injections given to a patient and recorded on the patient's hospital anaesthetic record card was noted and the patient's recovery room record was examined. At the end of the three-month evaluation period the physicians and the nurses independently answered questionnaires that had been prepared for them.

\section{RESULtS}

Details of the nurses, all of whom were female, are as follows. P 1, 33 years old 
TABLE I

\begin{tabular}{lr}
\hline Minor Gynaecological Operations & 116 \\
Major Gynaecological Operations & 82 \\
Miscellaneous Operations Involving Limbs, & \\
and Superficial Areas of Head, Neck and Trunk & 203 \\
Eye, Oral, and E.N.T. Operations & 27 \\
Minor Intra-abdominal Operations & 14 \\
Major Intra-abdominal Operations & 114 \\
Major Bone Operations & 24 \\
& Total \\
\hline
\end{tabular}

and single, had spent two years as a University student and worked as an uncertified nursing aide before taking her nurse's training, which she had just completed. $P 2,25$ years old and married had recently spent three years in general and orthopedic nursing. P 3, 21 years old and married, was a recent graduate. P 4, 21 years old and married had spent a year after graduation looking after post-partum mothers. During training the marks of two of them had been very slightly below the class average, one very slightly above it, and P 1 was 11.4 per cent in excess of it. The fifteen participating physicians varied in experience from recent specialists to veterans of the operating room.

The three-month training period presented no serious problems and the weekly meetings were terminated after six weeks. The nurses found working in rapid succession with different anaesthetists very confusing and this arrangement had to be changed. They also preferred an extremely didactic form of instruction.

During the three-month evaluation period five hundred and eighty patients became involved in the project. Details of the operations appear in Table I. Five hundred and forty-nine patients were in the ASA Status I and II risk category and almost all were adults.

\section{Summary of Information Collected During Supervision of Nurses}

Some analyses were done with a one way analysis of variance model for independent groups. If an overall significance was obtained at an alpha level of 0.05 then Schiffe's test for multiple comparisons was used to determine which pairs of means were the source of the significance. Dependent groups were tested on a one way analysis of variance model for repeated measures. If an overall significance was obtained at an alpha level of 0.05 then a series of dependent "t" tests were done to determine which pair of means was statistically different. The results of all such analyses are presented here.

A. The total evaluation period of three months was divided into three successive units of one month each (E 1, E 2, and E 3). A total of 540 operations were performed in which one of the four nurses was involved (i.e. 183, 219, and 147 operations for $\mathrm{E} 1$ to $\mathrm{E} 3$ respectively).

1. During each successive evaluation period statistically fewer casual visits per operation were made by the supervising anaesthetists. There were 1.68, 1.17, 0.82 casual visits per operation for $\mathrm{E} 1$ to $\mathrm{E} 3$ respectively, with an overall average of 1.21 casual visits per operation for the three months. 
2. On the other hand statistically more visits were requested by the nurses during the second evaluation period (E 2) than during E 1 or E 3 .

There were $0.49,0.76,0.46$ requested visits per operation for $\mathrm{E} 1$ to $\mathrm{E} 3$ respectively, with an overall average of 0.59 requested visits per operation for the three months.

B. Of the 549 operations, 226 entailed intra-abdominal surgery, 323 extra-abdominal surgery.

3. During the total evaluation period statistically more casual visits and more requested visits were made during intra-abdominal surgery than during extra-abdominal surgery.

a) intra-abdominal: 1.43 casual visits/operation and 0.92 requested visits/ operation

b) extra-abdominal: 1.05 casual visits/operation and 0.36 requested visits/ operation.

C. If the anaesthetist's visit was requested by a nurse one would predict the physician would assist or intervene; if the visit was casual, one would predict the anaesthetist would not intervene.

4. In 97.8 per cent of all requested visits the anaesthetist took some action.

5. In 6.5 per cent of all casual visits the anaesthetist took some action.

D. Some variations existed amongst the four nurses (P 1-P 4) in terms of casual visits.

6. Over the three-month evaluation period significantly more casual visits were made to $P 4$ than to $P 1, P 2$ or $P 3$; in addition $P 2$ received more casual visits than $P 1$.

7. There was also a significant difference amongst the nurses in the number of times action was taken following a casual visit: anaesthesia was modified more frequently whenever $\mathrm{P} 4$ was being supervised.

E. No variations were observed amongst the four nurses in terms of requested visits.

8. Over the three-month evaluation period of requests for the anaesthetist to come to the operating room.

F. There were no statistical differences amongst the four nurses in the frequency with which action was taken following requested visits.

The modifications of the anaesthesia made by the physician when action was taken during a casual visit involved depth of anaesthesia, cardiovascular or respiratory function, and muscle relaxation. On one occasion a severe and apparently unrecognized respiratory obstruction was corrected in a patient who was not intubated. No other situations requiring immediate treatment were ever found and usually nothing needed to be done. During the evaluation period the requested visits necessitated opinions about depth of anaesthesia, cardiovascular or respiratory function and muscle relaxation. An incidental approval of whatever course of action the nurse had already taken was often also solicited. The requested visits were never precipitated by an urgent problem. Once the patients had left the hospital the recovery room records were examined and the pattern of events had not differed from the one customary in this institution, so no statistical comparison was deemed necessary. 
TABLE II

The Number of Anaesthetists that Approved of Certain Categories of Patient and Surgical Procedure Being Supervised by the Nurses Compared With the Number of ANaEsthetists that did NOt APprove

\begin{tabular}{lccc}
\hline & & $\begin{array}{c}\text { Uncomplicated neuro-, } \\
\text { general surgical, and } \\
\text { gynaecological cases }\end{array}$ & $\begin{array}{c}\text { Thoracic, E.N.T., } \\
\text { oral, and plastic } \\
\text { cases of any kind }\end{array}$ \\
\hline Adults & ASA 1-2 & $10: 0$ & $10: 0$ \\
& ASA 3-4 & $4: 6$ & $3: 6$ \\
Children & ASA 1-2 & $10: 0$ & $10: 0$ \\
& ASA 3-4 & $2: 8$ & $1: 9$ \\
Infants & ASA 1-2 & $2: 8$ & $3: 7$ \\
& ASA 3-4 & $1: 9$ & $1: 9$ \\
\hline
\end{tabular}

During the course of the study 31 patients in an ASA category 3-4 were also supervised by nurses. No serious problems developed and the pattern of behavior of the persons concerned appears to have been similar to ASA 1 and 2 category patients.

\section{AnAETHETISTs' Questionnaire}

This questionnaire solicited opinions about the ability of the nurses and their potential to do this type of work, the changes in the work situation of the anaesthetist, and the interpersonal relations involved. Information from the ten anaesthetists who supervised anaesthesia for 35 to 70 patients during the evaluation period are presented here. Unanimity of opinion sometimes occurred but the extent of conflicting opinions is indicated by figures in the text. At some stage in the initial three months' training period all the anaesthetists instructed the nurses to administer certain drugs under certain specific circumstances, at the nurses' discretion. In other words, they were to assume immediate responsibility for the conduct of the anaesthesia. Thus the work being evaluated during the second three-month period involved assessing the patient's general condition, making decisions about whether to call a physician anaesthetist for guidance, or to carry out independent actions, which included the administration of various drugs.

None of the anaesthetists recalled any specific situation in which an independent decision by the nurse to administer a drug, or do anything, also endangered a patient's life. One anaesthetist recalled an event in which a failure to call him permitted a dangerous situation to develop, but the outcome was satisfactory. Two anaesthetists became concerned because some of the nurses (P 1, P 2 and P 4), gradually began taking independent action to an extent that worried them, and a third became apprehensive about $\mathrm{P} 4$.

It was clear that the nurses' capabilities increased with their operating room experience. The physician anaesthetists predictions of the nurses abilities to manage safely certain categories of cases once they had a year's training is summarized in Table II. Confidence was expressed regarding healthy adults and children but doubts existed about poor risk patients and most anaesthetists believed that their continued presence in the operating room would be necessary for all infant surgery 
and patients with severe cardiac problems or requiring resuscitation of any kind.

One crucial question in a study of this kind is whether the fact that the case would be supervised by a nurse influenced the choice of anaesthetic technique for any patient. Half the anaesthetists believed this was so, one stating that he intubated patients more often. Of greater significance, three of the anaesthetists considered that on rare occasions they had been faced with a conflict between what seemed to be the best anaesthetic technique for the particular surgery and patient and what seemed to be the most practical technique if the case was going to be under the immediate supervision of a nurse. This difficulty was resolved by that patient receiving the full attention of one particular anaesthetist, an alternative possible under the conditions of the project. This potential disruption of an operating room schedule could also be caused by two cases requiring induction or termination of anaesthesia at the same time, but this was not considered to be a potentially serious problem.

There can be no doubt that the anaesthetist assumes more professional responsibility when he supervises two patients simultaneously. Considering the final month of the evaluation period, supervising two nurses looking after adult ASA I and II patients undergoing orthopedic, general surgical or gynaecological operations was considered by four anaesthetists to contribute significantly to the stress of their day. Some anaesthetists (6:2) considered it made the work day more pleasant than if they had been by themselves in one operating room but others felt that the scope of the study did not really justify opinions on this aspect.

Some anaesthetists $(3: 7)$ thought that the introduction of nurses was a threat to their status, though another believed that it could only be so if the anaesthetist did not appear to be engaged in any useful activity at all. A gain in status was believed to be a possible outcome $(5: 5)$ but the reasons given were based on the appearance of the new work situation rather than the realities of it. Anxieties were expressed about the obvious pressures that were occasionally exerted on the nurse by the surgeon when the anaesthetist was not present because it was believed that such behaviour could have a detrimental effect on the patient's anaesthetic care.

\section{NURSES QuestionNAIRE}

The following observations are derived from a questionnaire distributed to the nurses at the end of the project. Three of them considered their nursing training a suitable background for their trial role and thought that it would take other personnel, except perhaps inhalation therapy technicians, longer to be trained for it. Whoever did it, they believed it essential that the person could tolerate stress, maintain good interpersonal relations and have some mechanical aptitude. They themselves were all strongly motivated to acquire a sound theoretical knowledge of anaesthesia, would have liked formal lectures during their training, and were dissatisfied with the responsibilities offered by the job in its original form. No major problems were experienced in getting along with the physician anaesthetists though their varying work patterns took time to learn. Once the other operating room nurses fully understood the role of the newcomers, good relations were established. The attitude of different surgeons ranged from rudeness, through an 
anxious scepticism, to overt enthusiasm, and ultimately they felt that they had been accepted into the scheme of things. It was the good relationship with the anaesthetists that had enabled them to alter their original role so that in most instances they assumed immediate responsibility for administering intravenous atropine, analgesics, barbiturates, and relaxant drugs without prior consultation. Their following comments refer to the job in this, its final form. The characteristics that appealed to them were making independent decisions about the patient's condition and drug requirements. They enjoyed the challenge, the sense of accomplishment, and the close professional relationship with physicians.

Two of them found the work tiring and another regretted the lack of patient contact as well as irregular and perhaps brief lunch breaks. Taking everything into consideration the work was largely unacceptable to them, and would only be done on a very temporary basis. Reference was made to the need for an association with poor risk patients and a greater variety of surgery, but whatever was done the job would have to be altered so that it was more interesting and offered more responsibility.

\section{Discussion}

During the interpretation of this study it is important to appreciate the circumstances under which it was carried out. The selection of anaesthetic technique and induction of anaesthesia was performed in a meticulous fashion by skilled anaesthetists. Only such individuals can consistently set up cases so that the ensuing anaesthetic course presents the minimum of problems. In addition, the frequency of casual visits noted on the protocols fails to indicate the real state of affairs. The nurses being supervised were in adjacent operating rooms and the supervising anaesthetist, in one operating room or in the hall-way occasionally looking through an operating room window. Thus, not only was there casual observation but also a physician was immediately available should his or her presence be requested. This illustrated one of the problems that developed. What was the supervising anaesthetist to do while cases were proceeding uneventfully? Perusing anaesthetic literature and doing simple investigations are obvious answers but this did not seem to be the complete answer and the anaesthetist appeared to be dangerously exposed to the hazards of introspection. It is an extension of this problem that in part determined the attitude of the physicians and the nurses to the work that they were doing. The acceptability to an individual of work being done depends on several factors, such as remuneration, working conditions, degree of stress, required motor activity and last but not least status or recognition by colleagues. It it clear that the nurses found the job deficient in at least some of these areas and some physicians would feel deprived if more of their work was delegated. In effect the nurses and physicians were competing for something of limited supply in the work situation of operating room anaesthetic practise. This situation could be ameliorated by other combinations of activities both within and outside the operating room, and clinical investigation, but success would depend on the resources of the individuals concerned and their environment. One comment at the end of the study was that the nurses selected were too intelligent for the job. However, though they may have had psychological traits that distinguished them from their 
peers during a brief interview, they did not seem to differ markedly from many of their peers throughout the hospital. In any case, to perform this kind of task with the maximum security for the patient the individual must be intelligent and have the capability and motivation for prolonged vigilance. These requirements were recognized by the four nurses and it was the overall character of the job and perhaps unrealistic expectations rather than its lack of responsibilities that caused them to reject it. Perhaps an important aspect of this situation is the number of other paramedical occupations that are becoming available to nurses. These often offer a wider range of diverse decisions and responsibilities in a more open environment than in the anaesthesia situation in which these nurses were working.

In conclusion, one object of this project was to determine whether recently graduated nurses could be trained in three months to look after certain anaesthetized patients safely. Information derived from the protocols and anaesthetists' questionnaires indicated that this was almost invariably so, but the information derived from the protocols could be interpreted in many ways. Such significance as it has lies in the fact that none of it was totally inconsistent with the opinions subsequently written by individual anaesthetists. However this report concerns a very small sample of nurses fulfilling a certain role in one institution after three months of instruction. The findings do not necessarily apply to other personnel in different institutions, or even in the same institution. Such interest as it may have lies in presentation of some of the problems that may arise if attempts are made to introduce this situation into a department previously fully staffed by physician anaesthetists. If anything deserves emphasis it is that safe clinical anaesthesia depends on the persons concerned having received adequate skilled training and supervised experience before assuming the responsibility for patients' lives. It was a deficiency in practical expertise and not a deficiency in theoretical knowledge that was responsible for the only hazardous episode in this study. If unnecessary morbidity and mortality are to be avoided, nurses must have adequate supervised practical training and, once this has been completed and they are doing routine work, a highly skilled physician anaesthetist must be available for that operating room within seconds of his or her presence being deemed necessary.

\section{SUMMARY}

Four recently graduated nurses received three months' training in the supervision of healthy adult surgical patients in whom surgical anaesthesia had been induced by specialist physician anaesthetists. During the course of this training the original project plan altered and the nurses assumed responsibility for administering atropine, opiates, barbiturates, and relaxant drugs. The training period was followed by a three months' evaluation period. One object of the project was to determine whether they could be trained in three months to look after certain anaesthetized patients safely and it appeared that this was almost invariably so. Conclusions from such a small sample must be viewed with great caution but it appears from independent questionnaires that each of the nurses found the work in its final form unrewarding and that if their work had been altered to make it acceptable to them, then some of the physician anaesthetists would themselves 
have been unable to obtain sufficient job satisfaction. It is emphasized that adequate practical instruction rather than theoretical knowledge is necessary for nonphysician personnel undertaking this type of work.

\section{RÉSUMÉ}

Quatre infirmières récemment graduées ont reçu une formation de trois mois pour faire la surveillance per-opératoire de patients adultes et en bonne santé. L'induction de l'anesthésie chez ces patients avait été pratiquée par des médecins spécialistes en anesthésiologie. Au cours de cette période de formation, le projet initial a été modifié de façon à confier aux infirmières la responsabilité d'administrer de l'atropine, des opiacés, des barbituriques et des curarisants. La session d'entraînement fut suivie d'une période d'évaluation d'une durée de trois mois. Un des buts du projet était de déterminer si un entraînement de trois mois permettait à des infirmières de prendre soin de façon sécuritaire de certains patients anesthésiés et il a semblé que c'était presqu'invariablement vrai. Les conclusions d'un si petit échantillonnage doivent toutefois être interprêtées avec prudence, mais un questionnaire indépendant semble démontrer que chacune des infirmières trouvait que dans sa forme finale, ce travail n'était pas gratifiant, et que si les tâches étaient modifiées à leur satisfaction, certains anesthésistes auraient alors été incapables de trouver suffisamment d'intérêt dans leur travail. Il est à noter qu'un enseignement pratique adéquat est plus utile que les connaissances théoriques pour un personnel paramédical devant œuvrer dans ce champ d'action.

\section{Participants in Project}

Nurses - B. Hainsworth, R.N., A. Stephenson, R.N., D. Yakimetz, R.N, and

D. Yoneda, R.N.

Doctors - G. Aitken, M.B., CH.B.; D.F. Cameron, M.D.; D. Dobson, M.B., CH.B.;

C.D. Elton, M.R.C.S., L.R.C.P.; E.A. Gain, M.D.; R.C. Gregg, M.D.;

J.M. Hagen, M.B., CH.B.; F.C. Haley, M.D.; H.G. Haynes, L.R.C.P. \& S.I.;

W.D. Kyle, M.D.; J.D. McFarland, M.B., CH.B.; J.W.R. McIntyre, M.R.C.S., L.R.C.P.;

G.T. Moonie, M.D.; D.R. Preston, M.D. and M. Rensaa, M.D.

Statistical Analysis - C.B. Hazlett, PH.D. 